\title{
Stereotactic body radiotherapy as a primary treatment for spinal metastasis: a single institution experience
}

\author{
Wui Ming (Eva) Yeung \\ Department of Clinical Oncology, Prince of Wales Hospital, Hong Kong, China \\ Correspondence to: Wui Ming (Eva) Yeung. Department of Clinical Oncology, Prince of Wales Hospital, Hong Kong, China. Email: ywm812@ha.org.hk.
}

\begin{abstract}
Background: To investigate the clinical outcome including efficacy and safety of stereotactic body radiotherapy (SBRT) in management of spinal metastasis.

Methods: Between 2010 and 2017, 26 patients with 32 metastatic spine lesions treated with SBRT were retrospectively reviewed. Local control (LC), overall survival (OS), prognostic factors and toxicity were analyzed.
\end{abstract}

Results: There were 15 female and 11 male patients (median age, 62 years; range 27 to 88 years). 32 metastatic spine lesions were treated with SBRT. The most frequent lesions treated were metastatic tumours of the lung ( $n=19)$, other primary histologies were prostate $(n=4)$, breast $(n=3)$, sarcoma $(n=2)$, hepatocellular carcinoma $(n=1)$, renal cell carcinoma $(n=1)$, stomach $(n=1)$, nasopharyngeal carcinoma $(n=1)$. Thoracic spine was the most common site treated $(n=18)$, followed by lumbosacral spine $(n=12)$ and cervical spine $(n=2)$. The most common dose fractionation used was $24 \mathrm{~Gy}$ in 3Fr. The median EQD2/10 Gy was 36 Gy (range, 34.7-59.7 Gy). The median planning target volume (PTV) was 50 cc (range, 10.4-194 cc). The median follow up was 23.4 months (range, 3-103 months). For the entire cohort, local tumour control (LC) at 1 year and 2 years were $83 \%$ and $71 \%$ respectively. The median OS was 30.6 months. The OS at 1 year and 2 years were $88 \%$ and $59 \%$ respectively. On univariate analysis for OS, presence of visceral metastases (HR 11.13, $\mathrm{P}=0.001)$, uncontrolled primary disease (HR 4.57, $\mathrm{P}=0.02)$ and presence of more than 3 vertebral metastasis (HR 5.50, $\mathrm{P}=0.04)$ were corelated with worst outcomes. On multivariate analysis for OS, only presence of visceral metastasis remained significant (HR 23.99, $\mathrm{P}=0.002)$. Acute toxicity mainly pain flare occurred in $16 \%$ of the treated lesions that can be managed with analgesics and steroid. Other adverse events were rare and no radiation induced myelopathy reported.

Conclusions: This study reports SBRT is a safe and effective treatment for spinal metastasis. Prognostic factors were identified to guide patient selection that would benefit from this treatment.

Keywords: Spinal metastasis; stereotactic body radiotherapy (SBRT); local control (LC); toxicity; prognostic factors

Submitted Apr 13, 2019. Accepted for publication Oct 28, 2020.

doi: 10.21037/apm-19-131

View this article at: http://dx.doi.org/10.21037/apm-19-131

\section{Introduction}

Patients with cancer are frequently affected by spinal metastases. Approximately $40 \%$ of cancer patients develop spinal metastasis. These metastases most commonly affect the thoracic spine $(70 \%)$, followed by the lumbar spine (20\%), cervical spine and sacrum respectively (1). The combination of an aging population at risk of developing cancer and the widespread availability of improved diagnostic imaging, such as magnetic resonance imaging (MRI) and 18F-labeled fluorodeoxyglucose positron emission tomography (PET), have increased the scope of this problem. Treatment is usually palliative, with the principle goals of pain relief, preservation of neurological function, and improvement in quality of life.

Conventional radiotherapy is widely used for pain palliation in spinal metastasis, because it is convenient and 
cost-effective considering the short-anticipated survival of metastatic cancer patients. The low radiation tolerance of the spinal cord and cauda equine limits optimal radiation dose delivery for durable tumour control. However, improvements in systemic therapies such as targeted therapies and immunotherapies are extending survival of many patients with cancer, requiring the treatment goals for metastatic spine tumors to shift from short-term palliation to long-term durable control.

Stereotactic body radiotherapy (SBRT) to the spine in a few fractions with image guidance allows the delivery of high doses of radiation to the tumor precisely with relative sparing of nearby critical structures, such as spinal cord. SBRT has been shown to be safe and effective treatment of spinal metastasis (2-7). Goals of SBRT are not only to palliate pain, but also to deliver ablative doses of RT to improve local control (LC) while maintaining the patient's functioning.

The aim of this study is to review our experience of using SBRT for spinal metastasis. We retrospectively assessed treatment outcomes and identified prognostic factors predicting LC and survival. The pain response as well as the toxicities of treatment were reviewed. We present the following article in accordance with the STROBE reporting checklist (available at http://dx.doi.org/10.21037/apm-19-131).

\section{Methods}

\section{Patient selection}

This is a retrospective study of 26 patients with 32 spinal metastatic lesions who were treated with SBRT during the period from 2010 to 2017. Patients with spinal metastasis who were treated with SBRT as post-operative adjuvant treatment or as re-irradiation due to previous treatment with conventional radiotherapy were excluded. Patient characteristics were extracted from paper records and clinical management system (CMS) of Hospital Authority, Hong Kong. Treatment details including immobilization technique, dose and fractionation, size of planning target volume and other dosimetric characteristics were reviewed from department record and Brain-Lab system. The pain relief was assessed by reviewing the symptom of pain before and after SBRT as well as the use of pain medications. Toxicities of the treatment were also reviewed.

\section{Radiotherapy technique and radiotherapy planning}

Treatment planning began with immobilization in the supine position using a customized ExacTrac mould. Computed tomography (CT)-based simulation was performed with intravenous contrast in $1.5 \mathrm{~mm}$-thick slices. MRI spine with T2W axial and sagittal study and T1W gadolinium contrast axial study was performed for treatment planning. Delineation of target and organ at risk (OAR) was conducted by co-registration of planning CT with MRI. Co-registration of planning CT with PET CT was also performed if available. For the contouring of gross tumour volume (GTV) and clinical target volume (CTV), we followed the International Spine Radiosurgery Consortium Consensus Guidelines for Target volume delineation in Spinal Stereotactic Radiosurgery since the publication of the guideline in 2012 (8). However, before the publication of the guideline in 2012, the GTV and CTV was defined at the discretion of treating oncologists. In principle, the GTV encompassed the lesion as visualized on pretreatment CT or MRI. The CTV encompassed the GTV and surrounding vertebral body and additional spinal structure deemed to be at risk for recurrence. The radiotherapy technique used is intensity modulated radiotherapy (IMRT) delivered with ExacTrac setup. We used Varian 2300iX Linac with add-on m3 MLC, add-on BrainLAB ExactTrac stereoscopic X-ray image guided (IGRT) system with automated Robotic 6D couch, and BrainLAB iPlanNet with iPlan RT Dose 4.5x stereotactic treatment planning system. The ExacTrac patient set up is based on real time $\mathrm{X}$-ray images with the patient on the couch. The system calculates the initial setup error from the image against the CT image used for treatment planning. The algorithm can detect the $6 \mathrm{D}$ errors up to $1 \mathrm{~mm}$ and 1-degre accuracy in all six degrees of freedom of setup. The automated Robotic couch relays to compensate for the setup errors. The mechanical correlation capability of the $6 \mathrm{D}$ couch was also found to be accurate up to $1 \mathrm{~mm}$ and 1 degree (9). Due to this exceptionally precise patient setup technique, the CTV-PTV margin used in our center is $1.5 \mathrm{~mm}$ isotropically. For the contouring of OAR, spinal cord was outlined using the T2W MRI if available, an additional of $1.5 \mathrm{~mm}$ margin from spinal cord defines spinal cord planning organ at risk volume (PRV). Other organ at risks were contoured including cauda equina, brachial plexus, esophagus, bowel and kidney etc.

\section{Follow up and statistical analysis}

There is no specific follow up protocol for patients after treatment with SBRT. However, patients were periodically monitored by physical and radiographic examination during 
their follow up for the treatment of primary cancer. Local progression was defined as local tumour progression by imaging (CT, MRI or FDG-PET CT).

Statistical analysis was done using SPSS with statistical package version 22. LC was calculated from the starting of radiotherapy until the date of progression or the date of last follow up. Overall survival (OS) was calculated from the date of starting of radiotherapy until the date of death or the date of last follow up. Estimates of OS were calculated using the Kaplan-Meier Method. The prognostic impact of parameters was assessed by univariate and multivariate cox regression methods. Hazard ratios (HR) and corresponding $95 \%$ CI were computed. A probability value $\leq 0.05$ was considered statistically significant.

\section{Research ethics}

This study was conducted in accordance with the Declaration of Helsinki (as revised in 2013). This study was approved by the Joint Chinese University of Hong KongNew Territories East Cluster Clinical Research Ethics Committee (CRE-2020.84).

\section{Results}

\section{Patient characteristics}

A total of 32 lesions in 26 patients were treated with SBRT from 2010 to 2017 . There were 15 female and 11 male patients (median age, 62 years; range 27 to 88 years). All patients presented with good performance prior to SBRT with Eastern Cooperative Oncology Group (ECOG) Performance Status $<2$. The most frequent lesions treated were metastatic tumours of the lung $(\mathrm{n}=19)$, other primary histologies were prostate $(n=4)$, breast $(n=3)$, sarcoma $(n=2)$, hepatocellular carcinoma $(n=1)$, renal cell carcinoma $(n=1)$, stomach $(\mathrm{n}=1)$, nasopharyngeal carcinoma $(\mathrm{n}=1)$. Thoracic spine $(56 \%)$ was the most common site treated, followed by lumbosacral spine (38\%) and cervical spine (7\%). The Spinal Instability Neoplastic Score (SINS) of the vertebral metastases were recorded. SINS (Table S1) is used to evaluate the degree of spinal instability in patients with spinal metastatic disease (10). In our patient cohort, $80 \%$ of vertebral metastases were stable with SINS under 6 and no lesion was classified as unstable (SINS $>13$ ). About half of the lesions treated were solitary spinal metastasis. At the time of SBRT, visceral metastasis was present in $38 \%$ of the treated lesions and $80 \%$ of cases has the primary disease under controlled. Majority of patients were asymptomatic from spinal metastasis as around $20 \%$ of patients presented with pain prior to SBRT. Less than $10 \%$ of lesions has epidural extension and paraspinal soft tissue extension. Table 1 summarized the patient characteristics and the features of the treated lesions.

\section{Dosimetry}

A total of 32 lesions were treated. 22 lesions were single level spinal metastasis and 11 lesions involved 2 vertebral levels. The most common dose fractionation used was 24 Gy in 3 fractions ( $\mathrm{n}=19)$, followed by 16 Gy in 1 fraction $(\mathrm{n}=7)$ and $27 \mathrm{~Gy}$ in 3 fractions $(\mathrm{n}=6)$. The prescription isodose ranged from $80 \%$ to $100 \%$. The median PTV volume was $50.1 \mathrm{cc}$. The median Conformity Index was 1.34 . The maximal point dose to spinal cord and PRV spinal cord were 18 Gy and 20 Gy respectively.

In order to correlate irradiation doses with clinical results, biological equivalent doses in 2 Gy fractions (EQD2) were calculated: an $\alpha / \beta$-ratio of 10 Gy was assumed for spinal metastasis and $\alpha / \beta$-ratio of 2 Gy for the spinal cord using the linear quadratic equation.

The median prescribed dose (EQD2/10 Gy) was 36 Gy. The median maximal point dose to spinal cord and PRV spinal cord (EQD2/2 Gy) were 36.9 Gy and 47.6 Gy respectively. Details of dosimetric characteristics are shown in Table 2.

\section{Clinical outcome}

The median follow up was 23.4 months (3-103 months). As for the 32 individual treated lesions, 11 lesions (34\%) in 9 patients developed local progression. The LC at 1-year and 2 -year was $83 \%$ and $71 \%$ respectively (Figure 1). For the 11 lesions with local progression, 2 local disease progressions were detected by MRI and the remaining 9 were detected by PET CT. The management of these local progression were using chemotherapy or targeted therapy for treatment of concurrent systemic disease progression (in 7 patients) and best supportive care (in 2 patients).

At the time of analysis of OS, 11 patients died. 7 patients died of disease progression, 2 patients died of sepsis, 1 patient died of pneumonia and 1 patient died of unknown cause that cannot be found from the medical record system. The median OS of the 26 patients was 30.6 months and the 1-year and 2-year OS was $88 \%$ and $59 \%$ respectively (Figure 2). 
Table 1 Patient $(n=26)$ and lesion $(n=32)$ characteristics

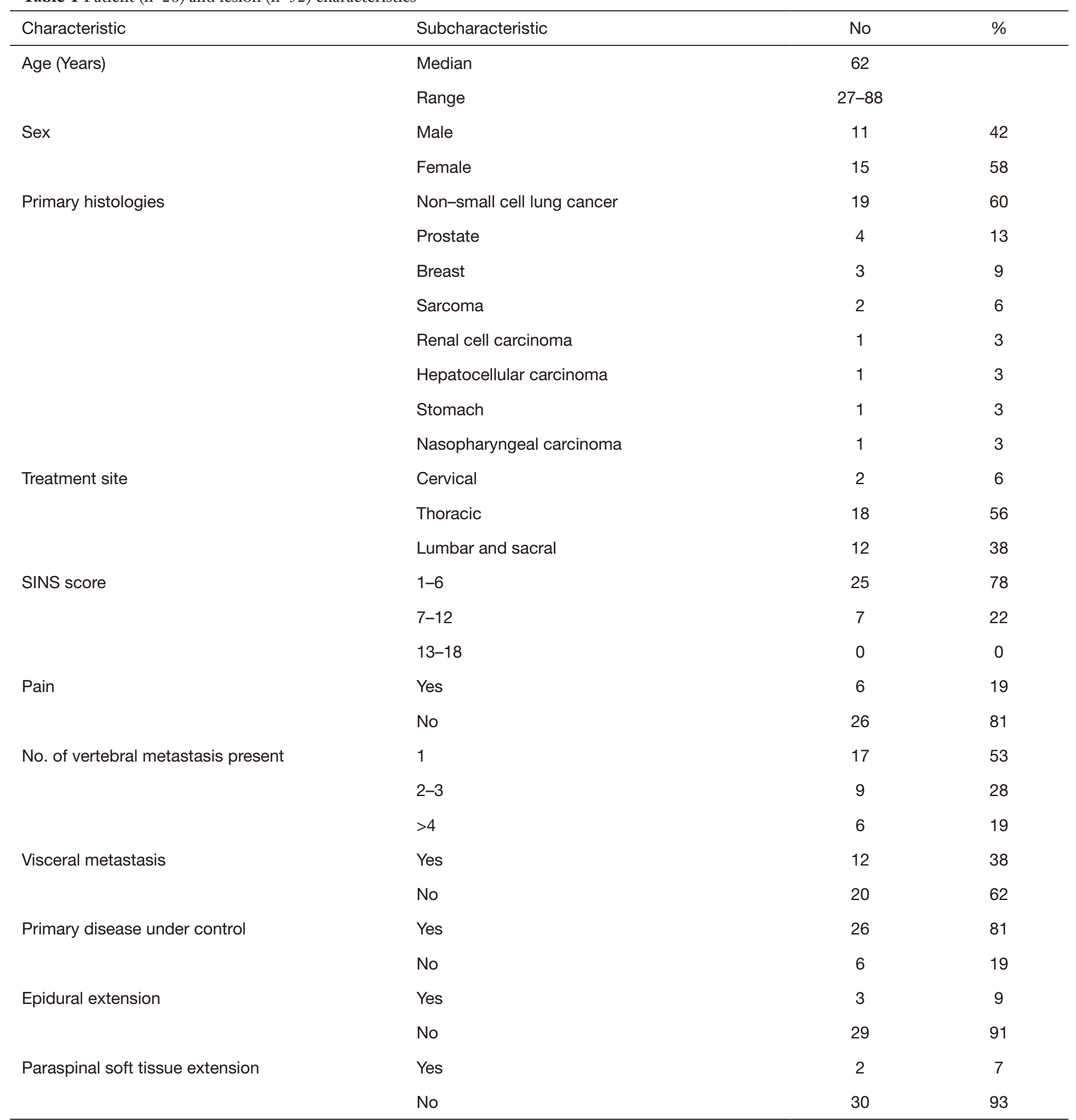

SINS, spinal Instability Neoplastic Score. 
Table 2 Dosimetric characteristics $(\mathrm{n}=32)$

\begin{tabular}{|c|c|c|}
\hline Characteristic & Subcharacteristic & Value \\
\hline Number of vertebrae treated in one target volume & 2 & 10 \\
\hline \multirow[t]{2}{*}{ Prescribed dose (Gy) } & 16 Gy × 1 & 7 \\
\hline & 8 Gy $\times 3$ & 19 \\
\hline \multirow[t]{2}{*}{ Prescribed dose (EQD2/10 Gy) } & Median & 36 \\
\hline & Range & $34.7-59.7$ \\
\hline \multirow[t]{2}{*}{ PTV volume $(\mathrm{cc})$} & Median & 50.1 \\
\hline & Range & $10.4-194.0$ \\
\hline \multirow[t]{2}{*}{ Maximal point dose spinal cord (EQD2/2 Gy) } & Median & 36.9 \\
\hline & Range & $26.2-52.9$ \\
\hline \multirow[t]{2}{*}{ Maximal point dose spinal cord PRV (Gy) } & Median & 20 \\
\hline & Range & $13.8-23.8$ \\
\hline \multirow[t]{2}{*}{ Maximal point dose spinal cord PRV (EQD2/2 Gy) } & Median & 47.6 \\
\hline & Range & $36-66.2$ \\
\hline \multirow[t]{2}{*}{ Conformity index } & Median & 1.34 \\
\hline & Range & $1.02-2.27$ \\
\hline
\end{tabular}

EDQ2, equivalent doses in 2 Gy fractions; PTV, planning target volume; PRV, planning organ at risk volume.

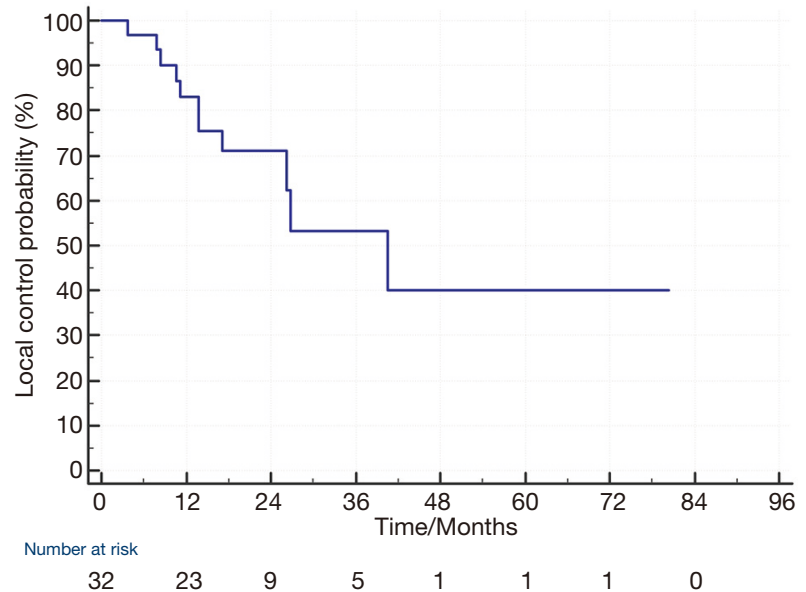

Figure 1 Local control of the 32 lesions.

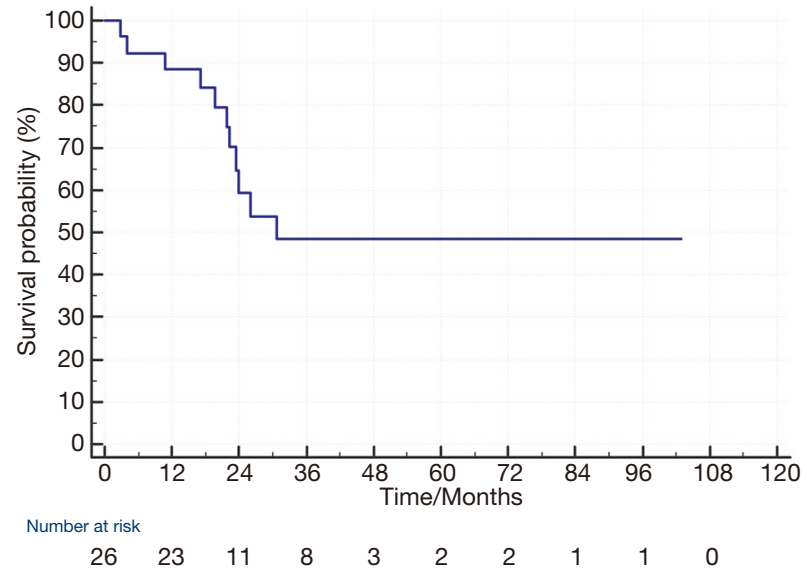

Figure 2 Kaplan-Meier estimates of overall survival of the 26 patients. 
Table 3 Univariate and multivariate analysis of factors on local control

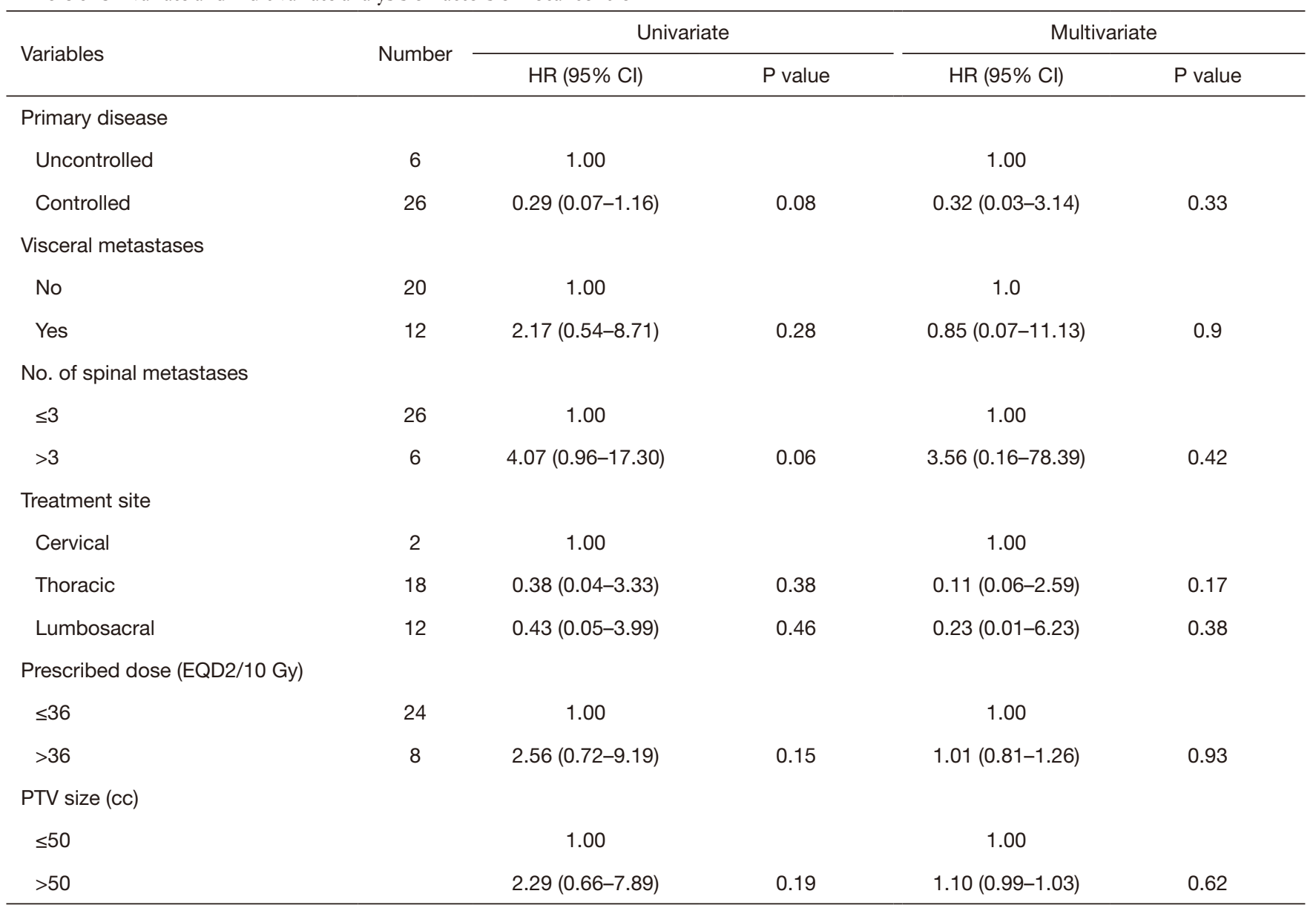

EDQ2, equivalent doses in 2 Gy fractions; PTV, planning target volume.

\section{Prognostic factors predicting $L C$}

Univariate and multivariate analysis were performed for patient and treatment factors associated with local tumour control. Patient factors including presence or absence of visceral metastasis, number of spinal metastasis, primary disease controlled or uncontrolled and treatment factors including treatment site, prescribed dose (in EQD2/10 Gy), PTV size were analyzed. None of the above factors has statistical significant effect on the LC (Table 3). However, there is a trend towards better LC in for the lesions with controlled primary disease $(\mathrm{P}=0.08)$ and at the presence of oligometastatic spine disease (equal to or less than 3 vertebral metastases), $(\mathrm{P}=0.06)$.

\section{Prognostic factors predicting $O S$}

On univariate analysis, several factors were found to have statistically significant adverse impact on OS. Patients with uncontrolled primary disease (HR 4.57, 95\% CI, 1.3315.71, $\mathrm{P}=0.02$ ), presence of visceral metastases (HR 11.13, 95\% CI, 2.78-44.52, $\mathrm{P}=0.001)$ and more than 3 vertebral metastases (HR 5.50, 95\% CI, 1.10-27.48, $\mathrm{P}=0.04$ ) had poor OS. On multivariate analysis, presence of visceral metastasis remained significantly associated with worst OS (HR 23.99, 95\% CI, 3.18-180.90, $\mathrm{P}=0.002)$. Age, sex and the presence of local failure of the treated lesion does not affect OS in univariate or multivariate analysis. Table 4 summarized the findings of univariate and multivariate analysis.

\section{Pain relief}

In our patient cohort, majority of patients were asymptomatic and only 6 patients (19\%) had painful spinal metastasis at the time of SBRT. 2 patients required strong opioids for pain 
Table 4 Univariate and multivariate analysis of factors on overall survival

\begin{tabular}{|c|c|c|c|c|c|}
\hline Variables & Number & \multicolumn{2}{|c|}{ Univariate } & \multicolumn{2}{|c|}{ Multivariate } \\
\hline \multicolumn{6}{|l|}{ Sex } \\
\hline Male & 11 & 1.00 & & 1.0 & \\
\hline Female & 15 & $0.74(0.22-2.44)$ & 0.62 & $0.96(0.22-4.26)$ & 0.96 \\
\hline$<60$ & 8 & 1.00 & & 1.0 & \\
\hline$\geq 60$ & 18 & $0.66(0.20-2.18)$ & 0.5 & $1.82(0.28-11.69)$ & 0.53 \\
\hline \multicolumn{6}{|l|}{ Primary disease } \\
\hline Controlled & 22 & 1.00 & & 1.0 & \\
\hline$\leq 3$ & 24 & 1.00 & & 1.00 & \\
\hline$>3$ & 2 & $5.50(1.10-27.48)$ & 0.04 & $0.35(0.02-5.09)$ & 0.44 \\
\hline \multicolumn{6}{|l|}{ Local failure } \\
\hline No & 17 & 1.00 & & 1.00 & \\
\hline Yes & 9 & $0.98(0.30-3.25)$ & 0.97 & $1.19(0.22-6.42)$ & 0.84 \\
\hline \multicolumn{6}{|c|}{ Visceral metastases } \\
\hline No & 19 & 1.00 & & 1.00 & \\
\hline Yes & 7 & $11.13(2.78-44.52)$ & 0.001 & 23.99 (3.18-180.90) & 0.002 \\
\hline
\end{tabular}

control and one of the patient had Numeric Rating Scale (NRS) score 10 while the other patient's pain score was not charted. 3 patients required weak opioids, one of them had NRS score 6 while the other 2 patient's pain score were not reported. 1 patient reported pain but did not require analgesic. There was no standardized pain assessment after SBRT. However, from the medical record systems and the pain medication records, 3 patients had improvement in pain control after SBRT. The patient with NRS score 10 had pain score reduced to 4 at 1 month after SBRT. Another 2 patients also had partial pain relief at around 2 months after SBRT with reduction of analgesic dose. 1 patient had persistent pain despite follow up imaging did not show local progression. 1 patient died at 3 months after SBRT and the pain response cannot be assessed. 1 patient's pain response was not reported.

\section{Toxicity}

In general, treatment was well tolerated. One patient had vomiting after treatment. 5 patients reported pain flare and 3 of them did not require treatment, 1 patient required steroid and 1 patient need escalation of analgesic to opioid. There was one case of vertebral compression fracture (VCF). This patient had carcinoma of lung with a spinal metastasis at C7 and had SBRT 24 Gy in three Fractions. Follow up MRI at 8 months after SBRT showed vertebral collapse without disease progression and the patient was asymptomatic. There was no radiation induced myelopathy reported.

\section{Discussion}

Over the past decade, there was a dramatic change in treatment paradigms of spinal metastasis due to the advancement in radiation therapy and development of systemic therapy. With the advancement of systemic therapy such as targeted therapy and immunotherapy, the OS of our patient has been significantly prolonged. The treatment 
Table 5 Result from selected series using spine SBRT as de novo treatment

\begin{tabular}{|c|c|c|c|c|c|c|}
\hline Author (Year) & $\begin{array}{c}\text { Tumour/ } \\
\text { Patient } \\
\text { treated }\end{array}$ & Histology & $\begin{array}{l}\text { Median } \\
\text { follow up } \\
\text { (Months) }\end{array}$ & Local control & Overall survival & Tumour Dose/No. of Fractions \\
\hline Sahgal (2009) (4) & $23 / 14$ & Mixed & 21 & $85 \%$ (1 yr); 69\% (2 yr) & Not reported & Median 24 Gy/3Fr \\
\hline Wang (2012) (11) & $166 / 149$ & Mixed & 16 & $81 \%(1 \mathrm{yr}) ; 72 \%(2 \mathrm{yr})$ & Median 23 months & $27-30 \mathrm{~Gy} / 3 \mathrm{Fr}$ \\
\hline Park (2015) (12) & $45 / 28$ & Mixed & 7.4 & $95 \%(1 \mathrm{yr})$ & $47 \%$ (1 yr); 28\% (2 yr) & Median 27 Gy/3Fr (18-35 Gy/1-5Fr) \\
\hline Folkert (2014) (13) & $120 / 88$ & Sarcoma & 12.3 & $87.9 \%(1 \mathrm{yr})$ & Median 16.9 months & Median 28.5 Gy/3-6Fr or 24 Gy/1Fr \\
\hline $\begin{array}{l}\text { Guckenberger } \\
(2013)(14)\end{array}$ & $387 / 301$ & Mixed & 11.8 & $90 \%(1 \mathrm{yr}) ; 84 \%(2 \mathrm{yr})$ & $65 \%$ (1 yr); 44\% (2 yr) & Median 24 Gy/3Fr (10-60 Gy/1-20Fr) \\
\hline Current series & $32 / 26$ & Mixed & 23.4 & $83 \%(1 \mathrm{yr}) ; 71 \%(2 \mathrm{yr})$ & $\begin{array}{l}\text { Median } 30.6 \text { months; } \\
88 \% \text { (1 yr); 59\% (2 yr) }\end{array}$ & 24-27 Gy/3Fr or $16 \mathrm{~Gy} / 1 \mathrm{Fr}$ \\
\hline
\end{tabular}

goals for metastatic spine tumours shifted from short term palliation to long term durable control. The most important change to these paradigms has been the integration of stereotactic body radiation therapy, allowing delivery of tumoricidal radiation doses with sparing of nearby organs at risk. It not only provides pain relief but also sustained LC to patients with spinal metastasis.

The present study reports our experience of SBRT for the treatment of de novo spinal metastasis that is in patient who have not received prior surgical intervention or radiotherapy. The results demonstrate excellent LC with the 1-year and 2-year LC of $83 \%$ and $71 \%$ respectively. Table 5 summarized the results of selected series and our current series are included for comparison.

Our results are comparable with other series that reported 1-year LC ranged from $81 \%$ to $95 \%$ and 2 -year LC ranged from $69 \%$ to $84 \%$. There is no prospective data demonstrating the optimal fractionation scheduled for spine SBRT. The most common dose-fractionation used in these previous series was $24-27 \mathrm{~Gy} / 3 \mathrm{Fr}$ which is also the most frequent used dose-fractionation in our patient cohort. Our patients also had mixed type of primary histologies including radioresistant histologies such as renal cell carcinoma and sarcoma. The excellent LC result proofs the benefit of the use of SBRT in these patients as the high biological effective dose can overcome the radioresistance.

We did not identify any prognostic factors affecting the
LC, including PTV size, prescribed dose, treatment site, presence or absence of visceral metastasis, number of vertebral metastasis or whether primary disease under controlled.

OS varies substantially between studies using SBRT for the treatment of vertebral metastasis, ranged from 15 to 23 months. This may be explained by the heterogeneity of the primary histology and the difference in patient selection criteria. A substantially longer OS of median 30.6 months was observed in our current study compared with the other series shown in Table 5 which were published few years ago. Our patient cohort also includes mixed type histologies but most of the primaries carry better prognosis. Among the 19 lesions of lung primary, 10 had Epidermal Growth Factor Receptor (EGFR) Exon 21 L858R mutation while 5 had EGFR Exon 19 deletion. All lesions of breast primary were estrogen receptor positive. With the emergence of the targeted therapy for non-small cell lung cancer such as the second-generation EGFR tyrosine kinase inhibitors and Cyclin D Kinase 4/6 inhibitors for estrogen receptor positive breast cancer over the past few years, the survival of lung cancer and breast cancer patients has been further prolonged. Since conventional radiotherapy achieves pain control and local control for a short duration, majority of the patients in this study were at risk for recurring pain if conventional radiotherapy would have been performed. It is important to find out prognostic factors for patient selection for spine SBRT to achieve a sustained pain 
control and improve progression free survival. In our study, univariate analysis identified several clinical parameters which were correlated with worse survival outcomes: uncontrolled primary disease, presence of more than 3 vertebral metastases and presence of visceral metastasis. On multivariate analysis, only presence of visceral metastasis associated with poor OS. Our results correlate with the findings from a multi-institutional analysis by Guckenberger et al. which includes the largest number of spinal metastatic lesions (14). Three hundred and eightyseven spinal metastases were treated with SBRT and the median follow up was 11.8 months. On multivariate analysis for OS, male sex, performance $<90$, presence of visceral metastases, uncontrolled systemic disease and $>1$ vertebra treated with SBRT were correlated with worse outcomes.

One interesting point to note in our study, the absence of local progression of spinal metastatic lesions did not affect OS as one might postulate that improved local control can give rise to longer survival. In our study, for those patients suffered from local progression of spine lesion, majority of them received systemic therapy such as chemotherapy and targeted therapy which helps to control the local as well as systemic disease progression.

With respect to pain control, spine SBRT is efficacious for symptom palliation, an important outcome for patients of reasonable prognosis where the goal is to prolong progression-free survival. Conventional radiotherapy is associated with partial pain relief rates of approximately $60 \%$, with complete pain response rate of $24 \%$ (17). On the other hand, spine SBRT literature has reported complete pain response rates as high as $46 \%$ to $92 \%(16,18-20)$. The duration of pain response is also longer with spine SBRT. The multi-institutional analysis by Guckenberger et al. found that $56.3 \%$ of patients suffering mild/moderate pain pre-SBRT were pain free at 11.5 months. Over $80 \%$ of patients suffering severe pain at baseline had only mild/ moderate pain or were pain free at 11.5 months (14). In our study, majority of patients did not suffer from pain due to the spinal metastasis. For those patients who were symptomatic, pain relief can also be achieved at 1 to 2 months post SBRT. Unfortunately, the retrospective nature of this study did not allow a longitudinal pain assessment in a systematic manner, so the degree of pain relief and the duration of pain relief cannot be fully reported.

Nowadays, with the increasing use of PET CT, there will be more patients with asymptomatic oligometastatic spinal metastasis being detected. Despite they are asymptomatic, achieving a better local control is also of paramount importance especially in patients with reasonable survival as progression of spinal metastasis can result in devastating complications such as vertebral collapse and even spinal cord compression.

For the toxicities of SBRT for spinal metastasis, in general the acute toxicities are mild and very limited in spine SBRT with $5 \%$ or less reported rates of severe and undesirable (grade 3 or higher) adverse events $(4,11,12,14)$. In our study, the toxicities encountered were mild and limited. The incidence of pain flare in our study was $16 \%$ (5 out of 32 treated lesions) and this can be managed with medication such as steroid and analgesics. In available literature, the incidence of pain flare reported was in the order of $23 \%$ to $68 \%(21,22)$. A dose-response relationship has been observed based on the pain flare analysis reported by Pan and colleagues that high-dose single-fraction SBRT yielded greater rates of pain flare than fractionated spine SBRT regimens (22). In our study, most of the spine lesions were treated with fractionated SBRT instead of single fraction which can explain the low incidence. And the single dose we used was 16 Gy which is not a very high dose. On the other hand, because of the retrospective nature of this study, the pain flare may be under-reported as we did not intend to look at this acute side effect.

For the late side effects, a more common late effect of spine SBRT is radiation-induced VCF. It is defined as a de novo fracture (i.e., a new endplate fracture or collapse deformity as compared with pre-treatment) or as progression of an existing fracture at the treatment site. Early institutional reports demonstrated crude risk of this complication from $11 \%$ to $39 \%$. A dose-complication relationship was observed in patient treated with $24 \mathrm{~Gy}$ in 1 fraction had nearly a $40 \%$ risk of VCF. Pre-existing risk factors should be considered in the overall management decision. SINS is useful tool for assessment as 3 out of 6 criteria including baseline VCF, lytic tumour and spinal deformity have been identified in the multi-institutional study to be significant predictors of VCF (22). A recent post hoc analysis of 2 prospective cohort confirms the utility of SINS as a predictive tool in this regard, demonstrating high SINS [7-12] as a statistical significant risk factors for VCF and symptomatic fractures (Hazard ratio 5.6 and 5.3 respectively) (23). In our study, $80 \%$ of the lesions treated were stable (SINS <6) and the SBRT dose used for single fraction SBRT was 16 Gy. Therefore, the incidence of VCF in our patient cohort was low as only 1 patient developed vertebral collapse after SBRT using $24 \mathrm{~Gy}$ in $3 \mathrm{Fr}$ and the SINS was 7 indicating there was pre-existing risk factors 
for VCF. Finally, radiation-induced myelopathy is the most feared late complication which can be serious and debilitating. There is no reported case of radiation-induced myelopathy in our study.

\section{Limitation of this study}

This study had the following limitations. Firstly, it was a retrospective study in a single institution with a small sample size. Secondly, there was no standard follow up imaging protocol and therefore the local control rate and the late toxicities such as vertebral compression fraction cannot be adequately evaluated. Besides, although the symptom of pain was reported but the severity was not evaluated using a standardized pain assessment such as numeric rating scale or even Brief Pain Inventory for a more comprehensive assessment. Therefore, the degree of pain relief or pain flare cannot be fully reported.

\section{Conclusions}

Our analysis showed SBRT as a primary treatment for spinal metastasis gives excellent local control with minimal toxicities. We identified prognostic factors associated with worse survival, including presence of visceral metastasis, uncontrolled primary disease and presence of more than 3 vertebral metastases. These factors should be considered for selecting a patient for spine SBRT. With the advent of standardized contouring guidelines, strict technical requirement for precise treatment delivery, and normal tissue tolerance guidelines for safe spinal cord practice, spine SBRT is currently regarded as a safe and effective technique. The clinical benefit of SBRT for carefully selected patients can be achieved through halting progression of disease, reducing patient symptoms and thus leading to improved functioning and quality of life.

\section{Acknowledgments}

I would like to thank Dr. Yvonne Yau, Dr Macy Tong and Dr Darren Poon for their support and guidance in this study. And special thanks to Mr Yu Kai Shing, Jimmy for the statistical support.

Funding: None.

\section{Footnote}

Provenance and Peer Review: This article was commissioned by the Guest Editors (Rebecca Yeung and Tai Chung Lam) for the series "Integrating Palliative Medicine in Oncology Care: The Hong Kong Experience" published in Annals of Palliative Medicine. The article has undergone external peer review.

Reporting Checklist: The author has completed the STROBE reporting checklist. Available at http://dx.doi.org/10.21037/ apm-19-131

Data Sharing Statement: Available at http://dx.doi. org/10.21037/apm-19-131

Conflicts of Interest: The author has completed the ICMJE uniform disclosure form (available at http://dx.doi. org/10.21037/apm-19-131). The series "Integration of Palliative Care into Oncology" was commissioned by the editorial office without any funding or sponsorship. The author has no other conflicts of interest to declare.

Ethical Statement: the authors are accountable for all aspects of the work in ensuring that questions related to the accuracy or integrity of any part of the work are appropriately investigated and resolved. This study was conducted in accordance with the Declaration of Helsinki (as revised in 2013). This study was approved by the Joint Chinese University of Hong Kong- New Territories East Cluster Clinical Research Ethics Committee (CRE2020.84). Informed consent was waived due to the retrospective nature of the study.

Open Access Statement: This is an Open Access article distributed in accordance with the Creative Commons Attribution-NonCommercial-NoDerivs 4.0 International License (CC BY-NC-ND 4.0), which permits the noncommercial replication and distribution of the article with the strict proviso that no changes or edits are made and the original work is properly cited (including links to both the formal publication through the relevant DOI and the license). See: https://creativecommons.org/licenses/by-nc-nd/4.0/.

\section{References}

1. Sciubba DM, Petteys RJ, Dekutoski MB, et al. Diagnosis and management of metastatic spine disease. A review. J Neurosurg Spine 2010;13:94-108.

2. Yamada Y, Bilsky MH, Lovelock DM, et al. Highdose, single-fraction image-guided intensity-modulated 
radiotherapy for metastatic spinal lesions. Int J Radiat Oncol Biol Phys 2008;71:484-90.

3. Nelson JW, Yoo DS, Sampson JH, et al. Stereotactic body radiotherapy for lesions of the spine and paraspinal regions. Int J Radiat Oncol Biol Phys 2009;73:1369-75.

4. Sahgal A, Ames C, Chou D, et al. Stereotactic body radiotherapy is effective salvage therapy for patients with prior radiation of spinal metastases. Int J Radiat Oncol Biol Phys 2009;74:723-31.

5. Mahadevan A, Floyd S, Wong E, et al. Stereotactic body radiotherapy reirradiation for recurrent epidural spinal metastases. Int J Radiat Oncol Biol Phys 2011;81:1500-5.

6. Ahmed KA, Stauder MC, Miller RC, et al. Stereotactic body radiation therapy in spinal metastases. Int J Radiat Oncol Biol Phys 2012;82:e803-9.

7. Sahgal A, Ma L, Weinberg V, et al. Reirradiation human spinal cord tolerance for stereotactic body radiotherapy. Int J Radiat Oncol Biol Phys 2012;82:107-16.

8. Cox BW, Spratt DE, Lovelock M, et al. International Spine Radiosurgery Consortium consensus guidelines for target volume definition in spinal stereotactic radiosurgery. Int J Radiat Oncol Biol Phys 2012;83:e597-605.

9. Yuen Kan Ngar D, Lok-Man Cheung M, Koon-Ming Kam M, et al. A novel compound 6D-offset simulating phantom and quality assurance program for stereotactic image-guided radiation therapy system. J Appl Clin Med Phys 2014;14:4297.

10. Fisher CG, DiPaola CP, Ryken TC, et al. A novel classification system for spinal instability in neoplastic disease: an evidence-based approach and expert consensus from the Spine Oncology Study Group. Spine (Phila Pa 1976) 2010;35:E1221-9.

11. Wang XS, Rhines LD, Shiu AS, et al. Stereotactic body radiation therapy for management of spinal metastases in patient without spinal cord compression: a phase 1-2 trial. Lancet Oncology 2012;13:395-402.

12. Park HJ, Kim HJ, Won JH, et al. Stereotactic Body Radiotherapy (SBRT) for Spinal Metastases: Who Will Benefit the Most from SBRT? Technol Cancer Res Treat 2015;14:159-67.

13. Folkert MR, Bilsky MH, Tom AK, et al. Outcomes and toxicity for hypofractionated and single-fraction image-guided stereotactic radiosurgery for sarcomas metastasizing to the spine. Int J Radiat Oncol Biol Phys 2014;88:1085-91.

14. Guckenberger M, Allgauer M, Appold S, et al. Safety and efficacy of stereotactic body radiotherapy for stage 1 non-small-cell lung cancer in routine clinical practice: a patterns-of-care and outcome analysis. J Thorac Oncol 2013;8:1050-8.

15. Sellin JN, Reichardt W, Bishop AJ, et al. Factors affecting survival in 37 consecutive patients undergoing de novo stereotactic radiosurgery for contiguous sites of vertebral body metastasis from renal cell carcinoma. J Neurosurg Spine 2015;22:52-9.

16. Anand AK, Venkadamanickam G, Punnakal AU, et al. Hypofractionated stereotactic body radiotherapy in spinal metastasis - with or without epidural extension. Clin Oncol (R Coll Radiol) 2015;27:345-52.

17. Chow E, Harris K, Fan G, et al. Palliative radiotherapy trials for bone metastases: a systematic review. J Clin Oncol 2007;25:1423-36.

18. Nguyen QN, Shiu AS, Rhines LD, et al. Management of spinal metastases from renal cell carcinoma using stereotactic body radiotherapy. Int J Radiat Oncol Biol Phys 2010;76:1185-92.

19. Ryu S, Jin R, Jin JY, et al. Pain control by image-guided radiosurgery for solitary spinal metastasis. J Pain Symptom Manage 2008;35:292-8.

20. Gerszten PC, Burton SA, Ozhasoglu C, et al. Radiosurgery for spinal metastases: clinical experience in 500 cases from a single institution. Spine (Phila Pa 1976) 2007;32:193-9.

21. Chiang A, Zeng L, Zhang L, et al. Pain flare is a common adverse event in steroid-naive patients after spine stereotactic body radiation therapy: a prospective clinical trial. Int J Radiat Oncol Biol Phys 2013;86:638-42.

22. Pan HY, Allen PK, Wang XS, et al. Incidence and predictive factors of pain flare after spine stereotactic body radiation therapy: secondary analysis of phase $1 / 2$ trials. Int J Radiat Oncol Biol Phys 2014;90:870-6.

23. Lee SH, Tatsui CE, Ghia AJ, et al. Can the spinal instability neoplastic score prior to spinal radiosurgery predict compression fractures following stereotactic spinal radiosurgery for metastatic spinal tumor?: a post hoc analysis of prospective phase II single-institution trials. J Neurooncol 2016;126:509-17.

Cite this article as: Yeung WM. Stereotactic body radiotherapy as a primary treatment for spinal metastasis: a single institution experience. Ann Palliat Med 2020;9(6):44674477. doi: 10.21037/apm-19-131 